\title{
Multidisciplinary approach in the clinical and laboratory investigation of a suspected case for anaplastic lymphoma associated with breast prosthesis
}

\author{
René Aloisio da Costa Vieira1,2,3* ๑, Idam de Oliveira-Junior ${ }^{1,2} \bullet$, Luciana da Fonseca Santos ${ }^{\oplus}$,

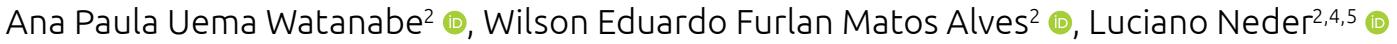

\section{ABSTRACT}

Introduction: Breast implant-associated anaplastic large cell lymphoma (BIA-ALCL) is a rare subtype of CD30-positive and ALKnegative (anaplastic lymphoma kinase) T cell lymphoma, which can develop in the pericapsular fibrous tissue and the late seromas around breast implants. If BIA-ALCL is suspected, an adequate diagnostic flow is essential. Materials and methods: A flowchart of the procedures performed in the diagnostic investigation is discussed, associating a clinical case, and conducting a review on the topic. Results: In the assessment of late and recurrent periprosthetic seromas, prior communication from the surgeon and the pathologist is essential, aiming at the adequate collection and storage of the aspirated material. The material must be promptly fractionated for microbiological assessment by culture, immediate or transoperative cytologic assessment, immunophenotyping by flow cytometry $(10 \mathrm{~mL})$, direct cytopathological examination, and obtaining cell block material $(50 \mathrm{~mL})$. For flow cytometry, the material must be sent fresh, $70 \%$ alcohol or $10 \%$ buffered formalin can be added for the other procedures. If it is impossible to send the aspirated fluid to the laboratory in less than six hours, it can be temporarily stored in a refrigerator at $4^{\circ} \mathrm{C}$. Immunophenotyping should be extensive, always assessing the expression of CD30 and ALK, regardless of cytological aspects. In cases of late and recurrent seromas in which BIA-ALCL is considered, even if initially discarded, it is suggested to perform capsulectomy with the removal of the prosthesis or careful clinical and laboratory monitoring. Conclusion: The diagnostic flowchart is essential, aiming at false-negative tests.

KEYWORDS: lymphoma, large cell, anaplastic; breast implants; lymphoma; seroma.

\section{INTRODUCTION}

Breast implant-associated anaplastic large cell lymphoma (BIA-ALCL) is a rare and indolent subtype of CD30-positive non-Hodgkin's lymphoma, primarily associated with breast implants, but which does not have translocations or expression of anaplastic lymphoma kinase (ALK) (ALK-negative ALCL). BIA-ALCLs are a subtype of $\mathrm{T}$ lymphoma that represents $10 \%$ of non-Hodgkin's lymphomas of the breast, which, in turn, correspond to $<1 \%$ of breast neoplasms ${ }^{1}$. The incidence of BIA-ALCL is 1 case for 500,000 to 3,000,000 women with late periprosthetic seroma.
Late periprosthetic seroma is a rare clinical entity, seen in less than $1 \%$ of cases with breast implants after one year ${ }^{2}$. Although the estimated individual risk for the development of seromas after textured implants is up to $10 \%^{3,4}$, the occurrence of late seromas is rare $(0.05 \%$ to $0.1 \%)$, and other differential diagnoses, such as trauma and infections, should be considered ${ }^{5,6}$.

The development of this subtype of $\mathrm{T}$ lymphoma is associated with, on average, 9 to 11 years after the placement of textured breast implants ${ }^{7-9}$. Long as this time may be, cases of BIAALCL have been described in up to two months, shortly after the replacement of breast implants. ${ }^{9}$. More recently, it has been

'School of Medicine of Botucatu - Botucatu (SP), Brazil.

${ }^{2}$ Barretos Cancer Hospital - Barretos (SP), Brazil.

${ }^{3}$ Muriaé Cancer Hospital - Muriaé (MG), Brazil.

${ }^{4}$ Ribeirão Preto Medical School, Universidade de São Paulo - Ribeirão Preto (SP), Brazil.

${ }^{5}$ Department of Pathology, Rede D'Or São Paulo - São Paulo (SP), Brazil.

*Corresponding author: reneacv@terra.com.br

Conflict of interests: nothing to declare.

Received on: 05/20/2020. Accepted on: 06/03/2020. 
proposed that the development of BIA-ALCL is associated with three main factors: textured breast implants, bacterial infection (biofilm), and genetic predisposition ${ }^{10}$.

Since the report of the first case, in $1997^{11}$, in a patient who had undergone cosmetic surgery for a breast implant, about 600 cases of BIA-ALCL have been described in the literature so far ${ }^{12}$. Immunophenotypically, BIA-ALCLs are indistinguishable from other anaplastic lymphomas of CD30-positive and ALK-negative $\mathrm{T}$ cells, and their diagnosis requires adequate clinical and laboratory assessment, which can be problematic in some cases. Some special care must be taken in the preservation of the material, which will be subjected to cytopathological analysis, immunohistochemistry assessment, and flow cytometry with immunophenotyping, which must include CD30 and ALK ${ }^{13-16}$. Therefore, a multidisciplinary approach and observance of a protocol of procedures are necessary to avoid the occurrence of false-negative results, a fact that motivated the present study.

\section{MATERIALS AND METHODS}

The study was approved by the Research Ethics Committee of Hospital do Câncer de Barretos, under No. 23026719.5.0000.5437/ 2019. An attempt was made to carry out a contextualized review on the topic, aiming to describe the procedure flowchart, the diagnostic steps, and the therapeutic care that must be performed by the mastologist. The diagnostic flowchart was exemplified using a suspected case of BIA-ALCL, in which extensive radiological and pathological assessment did not confirm the presence of this neoplasm.

\section{RESULTS}

A 42-year-old patient with bilateral additive mammoplasty for seven years and a history of late and recurrent seroma in the right breast associated with pruritus, sweating, and nocturnal chills for three weeks was submitted to assessment by mammography and breast ultrasound (BUS), showing locoregional axillary adenomegaly with cortical thickening, more significant on the right, and large ipsilateral periprosthetic collection (Figure 1).

Cytopathological assessment of the axillary lymph node and the right seroma was carried out by fine-needle aspiration, the results of which indicated a suspected lymphoma. Then, a radioguided excision of the right axillary lymph node was the procedure of choice, whose histopathological assessment showed only reactive lymphoid hyperplasia.

Subsequently, she underwent breast magnetic resonance imaging (MRI), which showed no mass or adenopathy, and positron emission tomography-computed tomography (PET-CT),
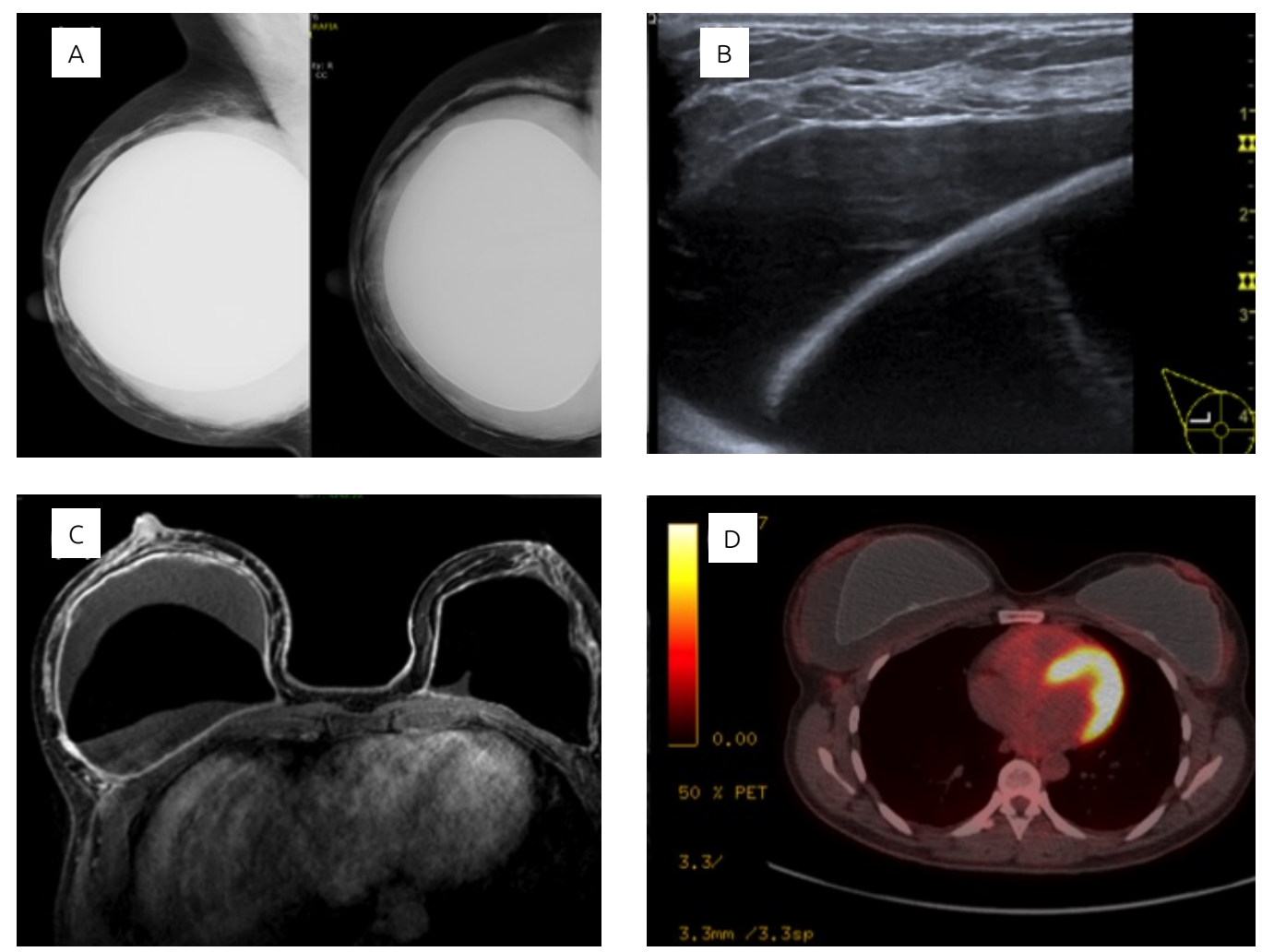

Figure 1. Negative radiological findings: (A) mammography; (B) breast ultrasound; (C) magnetic resonance; (D) positron emission computed tomography. 
which did not show any point of capture in the capsule or the axilla (Figure 1).

The patient underwent unilateral surgery, which consisted of total capsulectomy with the removal of the right prosthesis (Figure 2). During the surgical procedure, a direct cytological examination was carried out using cytospin smears of the aspirated fluid, with the suppurative and/or infectious process being discarded. Subsequently, separate sample syringes were collected for microbiological assessment by culture, $10 \mathrm{~mL}$ of the seroma for the flow cytometry exam, and $50 \mathrm{~mL}$ for the cytopathological exams and cell block immunohistochemistry.

Cytomorphological, microbiological, immunohistochemistry, and flow cytometry analyses ruled out lymphoma and infectious processes, showing only fibrosis and a mild reactive and polyclonal inflammatory cell infiltrate.

The patient progressed satisfactorily and was submitted to a new breast implant after four months.

\section{DISCUSSION}

The clinical presentation of BIA-ALCL is a collection of periprosthetic fluid (seroma) in $80 \%$ to $90 \%$ of cases, usually late and recurrent, as observed in the example case. Other presentations include breast swelling, asymmetry, pain, tumor mass around the implant, and local hyperemia ${ }^{7.8}$. The presentation as a tumor mass with lymph node involvement is rare, being observed in only $10 \%$ to $20 \%$ of patients, who may have cutaneous lesions, contraction of the implant capsule, and even B symptoms?

Once seroma is the main clinical manifestation, patients are usually initially assessed by BUS and submitted to aspiration of the fluid. In patients with a non-compliant mass or irregularities in the capsule, the diagnosis is facilitated by clinical suspicion and the possibility of performing core biopsy, but this situation is uncommon. Although BUS is the most used test in the initial assessment, in inconclusive cases, computed tomography (CT) or, preferably, MRI can be associated ${ }^{14}$ before considering the possibility of surgical treatment. PET-CT can be used in cases
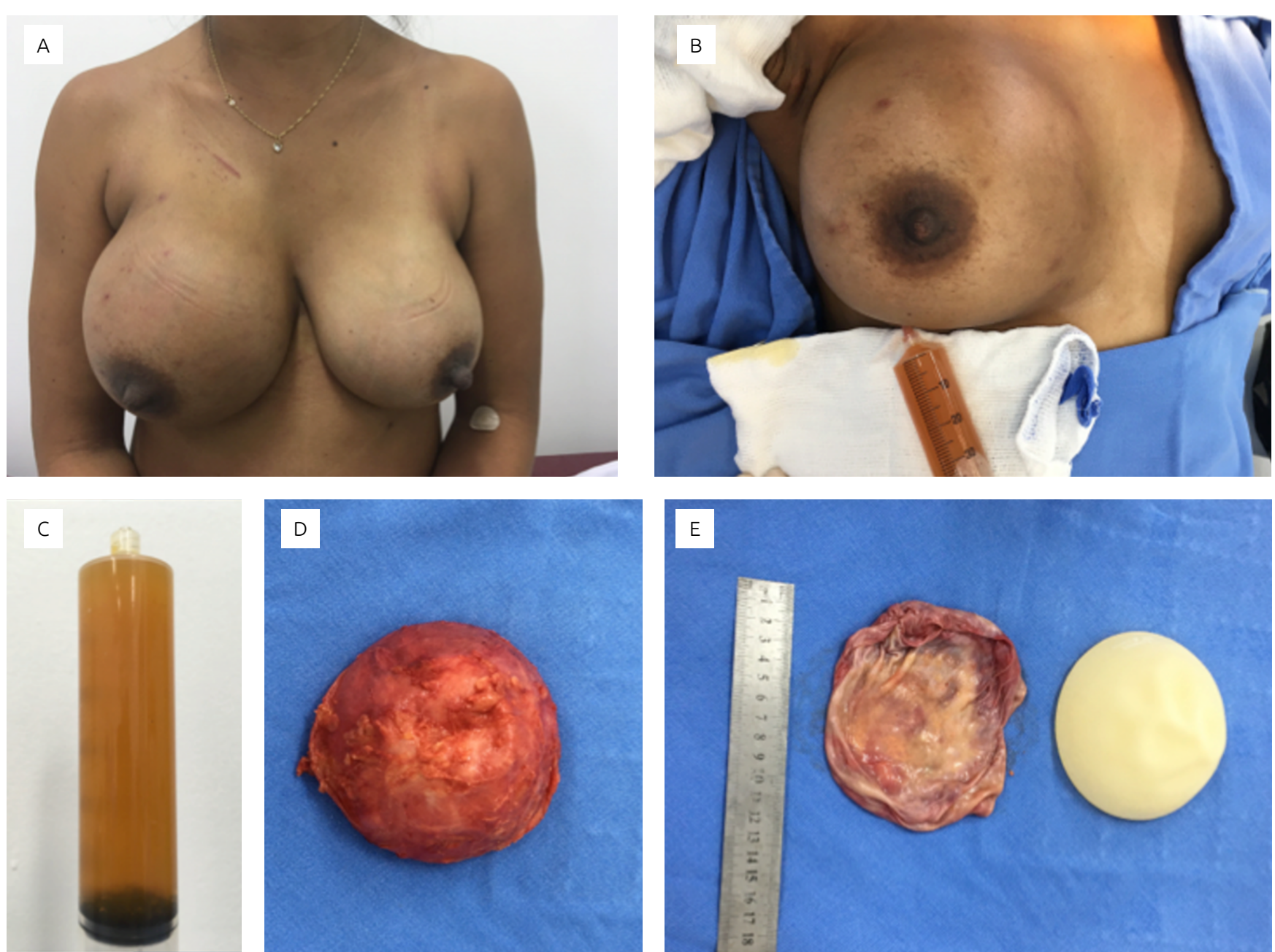

Figure 2. Clinical and surgical findings: (A) preoperative; (B) emptying of the seroma; (C) yellowish seroma; (D) total capsulectomy; (E) capsule without vegetation with the full textured prosthesis. 
with high clinical suspicion of malignancy, or even in confirmed cases of BIA-ALCL to improve staging.

In the diagnostic assessment before surgery, it is suggested to perform, whenever possible, the immunophenotyping of the periprosthetic fluid by flow cytometry. The cytological and immunophenotyping assessment of the seroma is very important since, in stage I, BIA-ALCL is confined to effusion ${ }^{3}$.

The sensitivities of BUS, CT, MRI, and PET-CT for infusion detection are $84 \%, 55 \%, 52 \%$, and $38 \%$, while for tumor mass sensitivities are $46 \%, 50 \%, 50 \%$, and $64 \%$, respectively ${ }^{17}$. Since the inflammatory process resulting from the surgical procedure can interfere with the results, PET-CT, if not performed before surgery, can be performed only after two to three months ${ }^{14}$. In the case presented, although the only radiological findings were associated with periprosthetic seroma, PET-CT showed no changes.

Some care is needed with the collected fluid to avoid falsenegative results. The aspiration puncture of the seroma with a cytological assessment on the same day is mandatory (less than six hours is considered adequate) to avoid cell degradation. If it is impossible to send the material to the laboratory in less than six hours, the material must be kept in a refrigerator at $4^{\circ} \mathrm{C}$ for up to 24 hours. In the presence of longer periods, the fluid must be discarded ${ }^{18}$, a fact that emphasizes the need to forward the material in the shortest possible time.

The pathologist must be informed in advance about the case, the date of the procedure, and the time that the material will be sent. It is suggested that no less than $50 \mathrm{~mL}$ of seroma be collected for cytopathological assessment and cell block preparation. At the same time, for flow cytometry immunophenotyping, it is recommended that at least $10 \mathrm{~mL}$ of aspirated fluid be collected in separate syringes.

The collected fluid can be viscous, serous, or hemorrhagic, when anticoagulant can be added, such as ethylenediaminetetraacetic acid or heparin. The fluid must be subjected to direct cytological assessment (Hematoxylin and Eosin stains, pap smear, Wright-Giemsa or May-Grünwald-Giemsa stain, according to the preference of the laboratory), immunohistochemical reactions in the cell block and immunophenotyping by flow cytometry, particularly to assess CD30 and ALK expression, regardless of morphological and cytological aspects.

There are several advantages in performing the cell block since the cytocentrifugation of the collected fluid makes it possible to obtain low-volume, high-cellularity, and paraffin-embedded material, which makes it possible to perform additional cuts and immunohistochemical reactions. The material can be sent without preservatives (in natura), or 70\% alcohol, methyl alcohol, or $10 \%$ buffered formalin can be added, depending on the preference of the laboratory ${ }^{18,19}$.

The minimum panel of antibodies used in flow cytometry must contain the anti-CD30, -CD163 and/or -CD68, -CD3, -CD20, -ALK, and pan-cytokeratin assessment, aiming to differentiate
BIA-ALCL from other B or T lymphomas, reactive macrophages, and carcinomas ${ }^{8,19}$. Classically, the diagnosis of BIA-ALCL is based on the detection, by flow cytometry, of CD30-positive and ALK-negative $\mathrm{T}$ lymphocytes in more than $10 \%$ of the cells in the aspirated fluid. For immunophenotyping, other markers can be used, such as CD5, CD2, CD7, CD43, CD4, CD8, granzyme B, and TIA $1^{18}$. However, Kadin et al. ${ }^{19}$ detected $>23 \%$ of CD30-positive $\mathrm{T}$ lymphocytes in late periprosthetic seroma in a 69-year-old patient. By investigating rearrangements of $\mathrm{T}$ cell antigenic receptors (TCRs), both in seroma and in peripheral blood, the authors concluded that these were activated $\mathrm{T}$ lymphocytes, which was consistent with local and peripheral immune responses, probably to bacterial superantigens that could be present in the biofilm formed on the surface of the prosthesis. These findings put into question the conception that the simple detection of $>10 \%$ of CD30-positive T lymphocytes in late seromas is sufficient for the diagnosis of BIA-ALCL, making it necessary, before closing the diagnosis, to employ a wide antibody panel and the joint assessment of immunohistochemical findings (cell block) and immunophenotyping by flow cytometry. Still, the investigation of TCR clonality and the assessment of mutations in the JAK1 and STAT3 genes can be of great help in doubtful cases?.

The presence of a previous infectious and/or inflammatory process is related to the development of seromas, which may be secondary to infections, trauma, or rupture of the prosthesis. As BIA-ALCL can be found in up to $10 \%$ of cases of late and recurrent seromas, it is plausible to consider the hypothesis that the malignant transformation occurs through the infiltration of inflammatory cells present in the seroma. Such a fact would justify the emptying of the seroma with the removal of the capsule and prosthesis in the late and recurrent seromas, as performed in the case analyzed in this study.

In the presence of evidence or highly suspected BIA-ALCL, the standard surgical procedure consists of emptying the periprosthetic content, capsulectomy, and removal of the breast prosthesis ${ }^{16}$, as performed in the present case. Generally, BIA-ALCL is confined to the fibrous capsule. However, it may present further infiltration ${ }^{3}$, with no indication of removal of the breast parenchyma. In the presence of a tumor mass, the concomitant resection of the tumor is suggested, with free margins ${ }^{20}$, since patients with complete resection present better outcome ${ }^{14}$.

Although the presence of bilateral disease occurs in only $4.6 \%$ of cases, in the presence of BIA-ALCL, bilateral implant and capsule surgery is suggested ${ }^{14}$. In cases of BIA-ALCL, the placement of a new prosthesis is discouraged ${ }^{20}$. However, when there is only diagnostic suspicion, the indication of bilaterality becomes questionable, and the surgeon must previously discuss this fact with the patient. In patients whose BIA-ALCL has not been confirmed, a new prosthesis may, in the future, be placed, as performed in the present case. 
About $20 \%$ of cases have metastatic lymph node disease so that in the absence of lymph node enlargement, lymph nodulectomy is not recommended, and there are no indications for the investigation of sentinel lymph node? ${ }^{7}$ Axillary lymphadenectomy has rarely been recommended, due to lymph node involvement bylymphoma ${ }^{14}$.

In patients with BIA-ALCL, the approach should be discussed in a multidisciplinary manner, with the participation of the mastologist and/or plastic surgeon, the hematologist, and the oncologist, with complete clinical staging, according to the tumor-nodule-metastasis system ${ }^{13,14}$. Adjuvant treatment is conducted with the team of clinical oncology or hematology, and the follow-up must be carried out, jointly, every three to six months in the first two years ${ }^{6}$. Adequate management of these patients is essential for therapeutic success.

\section{CONCLUSION}

BIA-ALCL is a rare subtype of non-Hodgkin's lymphoma with an indolent course, but which has been described with increasing frequency and associated with recurrent seromas with late development after the placement of textured breast implants. The establishment of a multidisciplinary approach with the observance of a clinical and laboratory investigation protocol is fundamental for the diagnostic resolution, the appropriate clinical management, and the reduction of false-negative cases.

\section{AUTHORS' CONTRIBUTIONS}

RACV: Conceptualization; Data curation; Formal analysis; Investigation; Supervision; Writing — original draft; Writing — review and editing.

IOJ: Conceptualization; Data curation; Investigation; Writing - review.

Santos LF: Data curation; Formal analysis; Investigation; Writing — original draft; Writing — review.

AUW: Data curation; Investigation; Writing — review.

LN: Conceptualization; Data curation; Formal analysis; Investigation; Writing — original draft; Writing — review.

\section{REFERENCES}

1. Aladily TN, Nathwani BN, Miranda RN, Kansal R, Yin CC, Protzel R, et al. Extranodal NK/T-cell lymphoma, nasal type, arising in association with saline breast implant: expanding the spectrum of breast implant-associated lymphomas. Am J Surg Pathol. 2012;36(11):1729-34. https://doi.org/10.1097/ pas.0b013e31826a006f

2. Ronchi A, Montella M, Argenzio V, Lucia A, De Renzo A, Alfano R, et al.Diagnosis of anaplastic large cell lymphoma on late periimplant breast seroma: Management of cytological sample by an integrated approach. Cytopathology. 2018;29(3):294-9. https://doi.org/10.1111/cyt.12541

3. Clemens MW, Medeiros LJ, Butler CE, Hunt KK, Fanale MA, Horwitz S, et al. Complete Surgical Excision Is Essential for the Management of Patients With Breast Implant-Associated Anaplastic Large-Cell Lymphoma. J Clin Oncol. 2016;34(2):1608. https://doi.org/10.1200/jco.2015.63.3412

4. Mehta-Shah N, Clemens MW, Horwitz SM. How I treat breast implant-associated anaplastic large cell lymphoma. Blood. 2018;132(18):1889-98. https://doi.org/10.1182/ blood-2018-03-785972

5. Clemens MW, Nava MB, Rocco N, Miranda RN. Understanding rare adverse sequelae of breast implants: anaplastic large-cell lymphoma, late seromas, and double capsules. Gland Surg. 2017;6(2):169-84. https://doi. org/10.21037/gs.2016.11.03

6. Di Napoli A, Pepe G, Giarnieri E, Cippitelli C, Bonifacino A, Mattei M, et al. Cytological diagnostic features of late breast implant seromas: From reactive to anaplastic large cell lymphoma. PLoS One. 2017;12(7):e0181097. https://doi. org/10.1371/journal.pone.0181097
7. Marra A, Viale G, Pileri SA, Pravettoni G, Viale G, De Lorenzi F, et al. Breast implant-associated anaplastic large cell lymphoma: A comprehensive review. Cancer Treat Rev. 2020;84:101963. https://doi.org/10.1016/j.ctrv.2020.101963

8. Quesada AE, Medeiros LJ, Clemens MW, Ferrufino-Schmidt MC, Pina-Oviedo S, Miranda RN. Breast implant-associated anaplastic large cell lymphoma: a review. Mod Pathol. 2019;32(2):166-88. https://doi.org/10.1038/s41379-018-0134-3

9. Ebner PJ, Liu A, Gould DJ, Patel KM. Breast implant-associated anaplastic large cell lymphoma, a systematic review and indepth evaluation of the current understanding. J Surg Oncol. 2019;120(4):573-7. https://doi.org/10.1002/jso.25626

10. Rastogi P, Deva AK, Prince HM. Breast Implant-Associated Anaplastic Large Cell Lymphoma. Curr Hematol Malig Rep. 2018;13(6):516-24. https://doi.org/10.1007/s11899-018-0478-2

11. Keech JA,Jr., Creech BJ. Anaplastic T-cell lymphoma in proximity to a saline-filled breast implant. Plast Reconstr Surg. 1997;100(2):5545. https://doi.org/10.1097/00006534-199708000-00065

12. Groth AK, Graf R. Breast Implant-Associated Anaplastic Large Cell Lymphoma (BIA-ALCL) and the Textured Breast Implant Crisis. Aesthetic Plast Surg. 2020;44(1):1-12. https:// doi.org/10.1007/s00266-019-01521-3

13. Laurent C, Haioun C, Brousset P, Gaulard P. New insights into breast implant-associated anaplastic large cell lymphoma. Curr Opin Oncol. 2018;30(5):292-300. https://doi.org/10.1097/cco.0000000000000476

14. Clemens MW, Jacobsen ED, Horwitz SM. 2019 NCCN Consensus Guidelines on the Diagnosis and Treatment of Breast ImplantAssociated Anaplastic Large Cell Lymphoma (BIA-ALCL). Aesthet Surg J. 2019;39(Supl. 1):S3-S13. https://doi.org/10.1093/asj/sjy331 
15. Clemens MW, Brody GS, Mahabir RC, Miranda RN. How to DiagnoseandTreatBreastImplant-AssociatedAnaplasticLarge Cell Lymphoma. Plast Reconstr Surg. 2018;141(4):586e-599e. https://doi.org/10.1097/prs.0000000000004262

16. Jones JL, Hanby AM, Wells C, Calaminici M, Johnson L, Turton P, et al. Breast implant-associated anaplastic large cell lymphoma (BIA-ALCL): an overview of presentation and pathogenesis and guidelines for pathological diagnosis and management. Histopathology. 2019;75(6):787-96. https://doi.org/10.1111/his.13932

17. Adrada BE, Miranda RN, Rauch GM, Arribas E, KanagalShamanna R, Clemens MW, et al. Breast implant-associated anaplastic large cell lymphoma: sensitivity, specificity, and findings of imaging studies in 44 patients. Breast Cancer Res Treat. 2014;147(1):1-14. https://doi.org/10.1007/s10549-014-3034-3
18. Di Napoli A. Achieving Reliable Diagnosis in Late Breast Implant Seromas: From Reactive to Anaplastic Large Cell Lymphoma. Plast Reconstr Surg. 2019;143(3S A Review of Breast Implant-Associated Anaplastic Large Cell Lymphoma):15S22S. https://doi.org/10.1097/prs.0000000000005565

19. Kadin EM, Morgan J, Xu H, Glicksman A. CD30+ T Cells in Late Seroma May Not Be Diagnostic of Breast ImplantAssociated Anaplastic Large Cell Lymphoma. Aesthet Surg J. 2017;37(7):771-775. http://doi.org/10.1093/asj/sjw286

20. Kaartinen I, Sunela K, Alanko J, Hukkinen K, KarjalainenLindsberg ML, Svarvar C. Breast implant-associated anaplastic large cell lymphoma - From diagnosis to treatment. Eur J Surg Oncol. 2017;43(8):1385-92. https://doi.org/10.1016/j. ejso.2017.05.021 